\title{
Experimental structure determination of the chemisorbed overlayers of chlorine and iodine on $A u\{111\}$
}

Article

Accepted Version

Zheleva, Z.V., Dhanak, V.R. and Held, G. (2010) Experimental structure determination of the chemisorbed overlayers of chlorine and iodine on Au\{111\}. Physical Chemistry Chemical Physics, 12 (36). pp. 10754-10758. ISSN 1463-9076 doi: https://doi.org/10.1039/c003461d Available at https://centaur.reading.ac.uk/15533/

It is advisable to refer to the publisher's version if you intend to cite from the work. See Guidance on citing.

To link to this article DOI: http://dx.doi.org/10.1039/c003461d

Publisher: Royal Society of Chemistry

All outputs in CentAUR are protected by Intellectual Property Rights law, including copyright law. Copyright and IPR is retained by the creators or other copyright holders. Terms and conditions for use of this material are defined in the End User Agreement.

www.reading.ac.uk/centaur 
Central Archive at the University of Reading

Reading's research outputs online 


\title{
Experimental structure determination of the chemisorbed overlayers of chlorine and iodine on $\mathrm{Au}\{111\}$
}

\author{
Zhasmina V. Zheleva, ${ }^{a}$ Vinod R. Dhanak, ${ }^{b}$ and Georg Held ${ }^{* a}$
}

\author{
May 11, 2010 \\ Received Xth $X X X X X X X X X X 20 X X$, Accepted Xth $X X X X X X X X X 20 X X$ \\ First published on the web Xth $X X X X X X X X X X 200 X$ \\ DOI: 10.1039/b000000x
}

\begin{abstract}
We have performed an experimental structure determination of the ordered $\mathrm{p}(\sqrt{3} \times \sqrt{3}) R 30^{\circ}$ structures of chlorine and iodine on $\mathrm{Au}\{111\}$ using low-energy electron diffraction (LEED). Despite great similarities in the structure of the underlying substrate, which shows only minor deviations from the bulk positions in both cases, chlorine and iodine are found to adsorb in different adsorption sites, fcc and hep hollow sites, respectively. The experimental Au-Cl and Au-I bond lengths of 2.56 and $2.84 \AA$ are close to the sums of the covalent radii supporting the view that the bond is essentially covalent in nature, however they are significantly shorter than predicted theoretically.
\end{abstract}

\section{Introduction}

It has been recognized recently that gold has great potential as heterogenous catalyst when the electronic structure of bulk gold is modified by reducing the particle size to a few $\mathrm{nm}$, by support interaction, or by electro-negative promoters. Examples are the low-temperature oxidation of $\mathrm{CO}$ and $\mathrm{CH}_{4}$ and the water-gas shift reaction on gold nanoparticles ${ }^{1-3}$, oxygenpromoted dissociation of $\mathrm{O}_{2}$ on $\mathrm{Au}\{111\}^{4}$, or chlorine-promoted selectivity in the oxidation of olefins and mercury $1,4,5$. The selectivity-enhancing effect of chlorine in particular and the general importance of halogens in electrochemical reactions have prompted a number of experimental and theoretical studies on halogen adsorption on the close packed $\{111\}$ surfaces of the group $1 \mathrm{~B}$ coinage metals $\mathrm{Cu}, \mathrm{Ag}$ and $\mathrm{Au}^{6-24}$.

Chemisorbed $\mathrm{Cl}$ layers display well-ordered phases, which make structure determinations possible with a number of experimental techniques. The experimental studies agree that $\mathrm{Cl}$ prefers fcc adsorption sites on $\mathrm{Cu}$ and $\mathrm{Ag}\{111\}^{9,10,17}$. Studies of iodine adsorption on $\mathrm{Cu}$ and $\mathrm{Ag}\{111\}$ indicate that iodine prefers fcc sites at low coverage, whereas at higher coverage compressed structures are found with both types of three-fold hollow sites, fcc and hcp, occupied ${ }^{6,8}$. Theoretical studies of halogen adsorption on transition metal surfaces usually find fcc sites as the lowest energy adsorption sites, however there are only small differences between hcp an fcc sites ${ }^{15-17,19}$.

Previous studies of chlorine adsorption on $\mathrm{Au}\{111\}$ have shown contradictory results. Spencer and Lambert ${ }^{7}$ concluded

a Department of Chemistry, The University of Reading, Whiteknights, Reading,UKE-mail: g.held@reading.ac.uk.

${ }^{b}$ Department of Physics, University of Liverpool, University Square, Liverpool, $U K$. on the basis of thermal desorption experiments that a layer of surface chloride $\left(\mathrm{AuCl}_{3}\right)$ was formed immediately upon chlorine adsorption on $\mathrm{Au}\{111\}$. Kastanas and Koel ${ }^{12}$ observed a $p(\sqrt{3} \times \sqrt{3}) R 30^{\circ}$ pattern in low-energy electron diffraction (LEED) below $230 \mathrm{~K}$ and found no evidence of chloride formation by X-ray photoelectron spectroscopy (XPS). They concluded that a chemisorbed chlorine overlayer was present with four $\mathrm{Cl}$ atoms per surface unit cell, which corresponds to a coverage of $1.33 \mathrm{ML}$. A more recent series of studies by Friend et al. combining scanning tunneling microscopy (STM), XPS and ab initio DFT calculations showed that the $p(\sqrt{3} \times \sqrt{3}) R 30^{\circ}$ superstructure has a much lower coverage of $0.33 \mathrm{ML}$. At this coverage the calculations predict the lowest energy for adsorption on fcc hollow sites in an undisturbed $\{111\}$ surface, however other structures, including hcp and bridge sites as well as a $\mathrm{Au}$ vacancy structure, are only insignificantly higher in energy $(\leq 0.1 \mathrm{eV})$. At a coverage above $0.33 \mathrm{ML}$ incorporation of $\mathrm{Au}$ atoms into the $\mathrm{Cl}$ layer is clearly favored $^{21,24}$. Theoretical electron density maps indicate that the $\mathrm{Au}-\mathrm{Cl}$ bond is predominantly covalent ${ }^{22,23}$. The present study is the first experimental proof of a covalently bound chemisorbed $\mathrm{Cl}$ layer leaving the underlying Au substrate in an essentially undisturbed bulk-like configuration.

Cochran and Farrell and Huang et al. examined the adsorption of iodine on $\mathrm{Au}\{111\}$ using a variety of methods ${ }^{14,25}$. They observed a series of ordered structures as a function of coverage. The diffraction pattern for the ordered layer with the lowest coverage was $p(\sqrt{3} \times \sqrt{3}) R 30^{\circ}$, corresponding to $0.33 \mathrm{ML}$, followed by uniaxially compressed $(p \times \sqrt{3})$ structures and a close packed layer with a Moire-type periodicity at saturation. In-air STM experiments showed the same $p(\sqrt{3} \times \sqrt{3}) R 30^{\circ}$ structure as observed in ultra high vacuum 
(UHV) at low coverage ${ }^{11}$, whereas X-ray diffraction of electrochemically deposited iodine only showed incommensurate monolayer phases similar to the saturated layer prepared in $\mathrm{UHV}^{13}$. The present LEED I-V structural study provides a detailed experimental structure determination of the $\mathrm{Au}\{111\}$ $p(\sqrt{3} \times \sqrt{3}) R 30^{\circ}$-I overlayer. In contrast to $\mathrm{Cl}$ and a recent theoretical study by Migani and Illas ${ }^{19}$, the atoms adsorb on hcp hollow sites with an essentially bulk-like substrate.
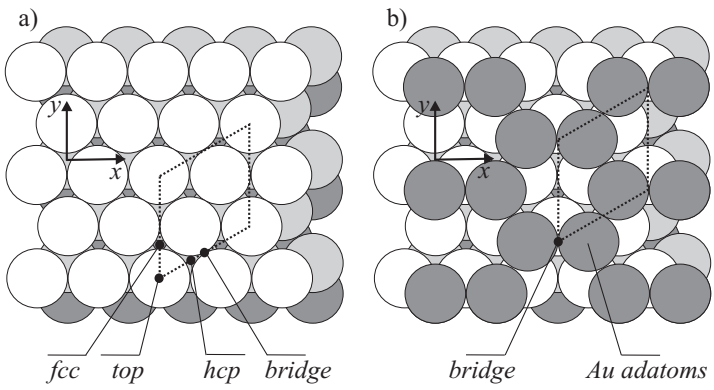

Fig. 1 Different geometry models tested in the LEED-IV data structure determination. Left: flat $\mathrm{Au}\{111\}$ substrate with atop, bridge, hcp and fcc sites indicated; right: adsorption on the bridge sites of a $\mathrm{Au}\{111\}$ surface covered with $0.67 \mathrm{ML}$ Au adatoms (0.33 ML vacancies).

\section{Experimental and Calculational Methods}

\subsection{Experiment}

The experiments were carried out in a standard UHV chamber equipped with a rear-view LEED optics (OCI Vacuum Microengineering) and an electron energy analyzer with dual anode X-ray source (PSP Vacuum Technology) for sample characterization by XPS. The base pressure of the system was below $2 \times 10^{-10}$ mbar, with hydrogen as the main residual gas in the chamber. The $\mathrm{Au}\{111\}$ surface was cleaned by cycles of $\mathrm{Ar}$ ion bombardment and annealing to approximately $800 \mathrm{~K}$. The sample was considered clean when no traces of contamination were found in XPS and the LEED pattern showed sharp integer order spots together with the satellite spots indicating the typical herringbone reconstruction of clean $\mathrm{Au}\{111\}$. Chlorine was generated in-situ from a solid state electrochemical cell and was dosed onto the clean surface at room temperature until a sharp $p(\sqrt{3} \times \sqrt{3}) R 30^{\circ}$ LEED pattern was obtained. Iodine was dosed from the gas phase using flakes of solid iodine placed in a glass ampoule, which was attached to the gas-dosing line of the UHV chamber. It was possible to observe the LEED pattern while iodine was dosed from the back- ground until a $p(\sqrt{3} \times \sqrt{3}) R 30^{\circ}$ superstructure was obtained and no more satellite spots from the herringbone reconstruction could be observed. Once the iodine or chlorine structures were formed, the sample was cooled to approximately $150 \mathrm{~K}$ for the LEED measurements. It was found that cooling enhanced the contrast and sharpened the spots in the LEED patterns. The spot intensities were measured at normal incidence as a function of the energy of the incident electrons (LEED-IV spectra). The LEED-IV spectra were background-subtracted and normalized with respect to the primary beam current. A total of 42 spots were extracted for both structures. The intensities of the symmetrically equivalent spots were averaged in order to reduce the experimental noise, which led to a cumulative energy range of inequivalent IV curves of $1799 \mathrm{eV}$ for the $\mathrm{Cl}$ and $1670 \mathrm{eV}$ for the I adsorption structure.

\subsection{LEED calculation}

The structure determination was performed with our fully automated "CLEED" program ${ }^{26}$ package based on fully dynamical scattering theory described by Pendry ${ }^{27}$ and Van Hove and Tong ${ }^{28}$. Bulk diffraction was calculated using Pendry's layer doubling method for gold bulk inter-layer spacings of $2.356 \AA$. Scattering phase shifts for $\mathrm{Au}, \mathrm{Cl}$ and I atoms were calculated as a function of energy using the program package provided by Barbieri and Van Hove ${ }^{29}$. The maximum angular momentum quantum number $l_{\max }$ was set to 10 . The imaginary and real parts of the inner potential were set to $4.0 \mathrm{eV}$ and $-5.5 \mathrm{eV}$, respectively, for the chlorine modified $\mathrm{Au}\{111\}$ surface and $4.0 \mathrm{eV}$ and $-8.5 \mathrm{eV}$ for the iodine modified $\mathrm{Au}\{111\}$ surface. Initially, the radial root mean square displacement for $\mathrm{Au}, \mathrm{Cl}$, and I were assumed $0.07 \AA, 0.12 \AA$, and $0.09 \AA$, respectively. In the final stage of the searches the displacements were optimized together with the inner potential to obtain the best fit between theory and experiment; these values are listed in Table 2. The downhill simplex method was used for the structure optimization ${ }^{30}$. The agreement between the experimental and theoretical IV curves was quantified with Pendry's $R_{P}$ factor and the error limits for the determined parameters were calculated using the $R R$ factor method ${ }^{31}$, which leads to a statistical error margin of $13 \%$ and $14 \%$ for the $\mathrm{Cl}$ and I data. Threefold rotational symmetry was assumed during the structural optimization for fcc, hcp, and top sites. For the bridge sites the only symmetry element was a mirror plane. The observed threefold symmetry is the result of the superposition of three domains rotated by $120^{\circ}$. The angle of incidence was optimized as part of the structural optimization, which increases the number of search parameters by 2 . 


\section{Results and Discussion}

For both the chlorine and iodine $p(\sqrt{3} \times \sqrt{3}) R 30^{\circ}$ overlayers, five types of structures were tested, in which the halogen atoms occupy atop, bridge, and threefold fcc or hcp hollow sites on a flat $\mathrm{Au}\{111\}$ surface (Fig. 1.a) and bridge sites on top of a layer of 0.67 ML Au adatoms (Fig. 1.b). These include all the structures that were found to be energetically most favorable for $0.33 \mathrm{ML} \mathrm{Cl}$ on $\mathrm{Au}\{111\}$ in a recent theoretical study ${ }^{21,24}$. A coverage of $0.33 \mathrm{ML}$ was assumed for all structure models such that the $(\sqrt{3} \times \sqrt{3})$ surface unit cell contains one halogen atom. The start geometry for each structure optimization was an ideal bulk-terminated substrate with the halogen atoms at a height that corresponds to hard spheres placed on the respective adsorption sites using the covalent radii of $0.99 \AA$ for $\mathrm{Cl}, 1.35 \AA$ for I and $1.44 \AA$ for $\mathrm{Au}$. During the structure optimization the atoms of the $\mathrm{Cl} / \mathrm{I}$ layer and the first three Au layers were allowed to relax in lateral and vertical direction. The lowest $R_{P}$ factors achieved for each type of structure are listed in Table 1.

\begin{tabular}{|l|c|c|}
\hline & $\mathrm{Cl}$ & $\mathrm{I}$ \\
\hline \multicolumn{3}{|c|}{ flat $\mathrm{Au}\{111\}$ substrate } \\
\hline fcc & $\mathbf{0 . 1 9}$ & 0.65 \\
hcp & 0.65 & $\mathbf{0 . 2 6}$ \\
top & 0.76 & 0.72 \\
bridge & 0.68 & 0.67 \\
\hline \multicolumn{3}{|c|}{$\mathrm{Au}\{111\}+0.67 \mathrm{ML} \mathrm{Au}$ adatoms } \\
\hline bridge & 0.66 & 0.67 \\
\hline
\end{tabular}

Table 1 The lowest $R_{P}$ factors achieved for $0.33 \mathrm{ML} \mathrm{Cl}$ and I on $\mathrm{Au}\{111\}$ in different $p(\sqrt{3} \times \sqrt{3}) R 30^{\circ}$ test geometries. See Figure 1 and text for further explanation.

For chlorine the $R_{P}$ value of 0.19 clearly identifies fcc as the adsorption site. The experimental and calculated IV curves for the optimized structure are shown in Figure 2 to illustrate the level of agreement between experiment and theoretical calculations. Both the numerical and visual agreement is within the range of recent LEED-IV studies for other atomic adsorbates on close-packed metal surfaces (see e.g. ${ }^{32-34}$ ). The parameters of the best fit structure are summarized in Table 2 and illustrated in Figure 3.

The relaxation of the three uppermost Au layers with respect to their bulk positions $\left(\Delta z_{n, n+1}\right)$ indicate small contractions of the inter-layer distances of less than $0.03 \AA$ or $1.2 \%$. The chlorine adatoms are at a vertical distance $d_{C l, 1}=1.94 \AA$ above the three-fold fcc hollow sites with identical chlorinegold bond lengths of $2.56 \AA$. This bond length is $0.32 / 0.22 \AA$ larger than the $\mathrm{Cl}$-Au bond lengths in $\mathrm{AuCl}_{3}\left(2.24 / 2.34 \AA^{35}\right)$ and $0.13 \AA$ larger than the sum of the covalent radii of gold and chlorine (1.44 $\AA$ and $0.99 \AA$ ). Ab initio calculations predict

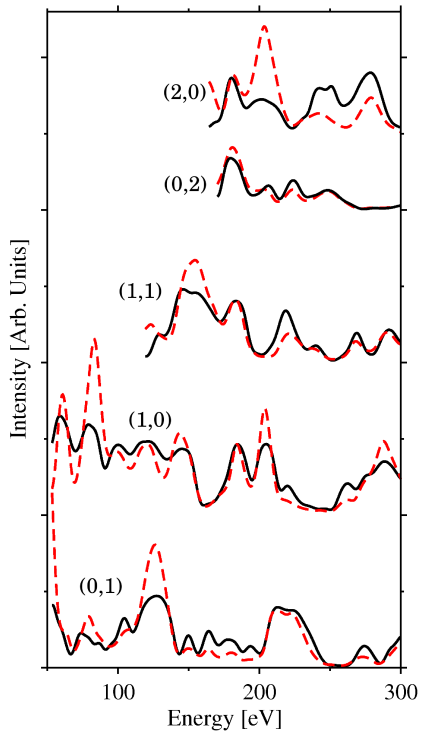

(a)

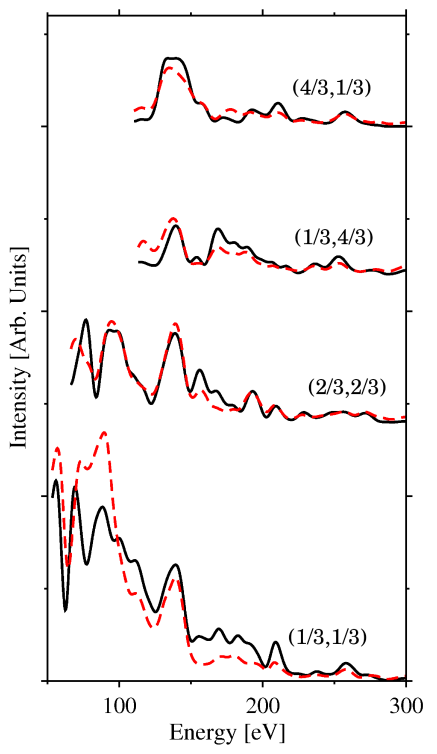

Fig. 2 Experimental (solid) and theoretical (dashed) LEED-IV curves for the best fit $\mathrm{Au}\{111\}-p(\sqrt{3} \times \sqrt{3}) R 30^{\circ}-\mathrm{Cl}$ structure: (a) integer (b) fractional order spots.

larger Cl-Au bond lengths of $2.69 \AA^{22}$ and $2.70 \AA^{19}$. Based on the electron density distribution, Baker et al. concluded that the $\mathrm{Au}-\mathrm{Cl}$ bond is predominantly covalent in nature ${ }^{22}$, which is in line with the common view of the bond nature of gold-(III)-chloride, $\mathrm{AuCl}_{3}$, because of the high electronegativity of gold ${ }^{35}$. The gold atoms in the first layer surrounding the fcc site move laterally slightly away from the $\mathrm{Cl}$ atom, $0.05 \pm 0.04 \AA$ with respect to their bulk positions.

Unlike chlorine, iodine atoms adsorb in hcp hollow sites. 


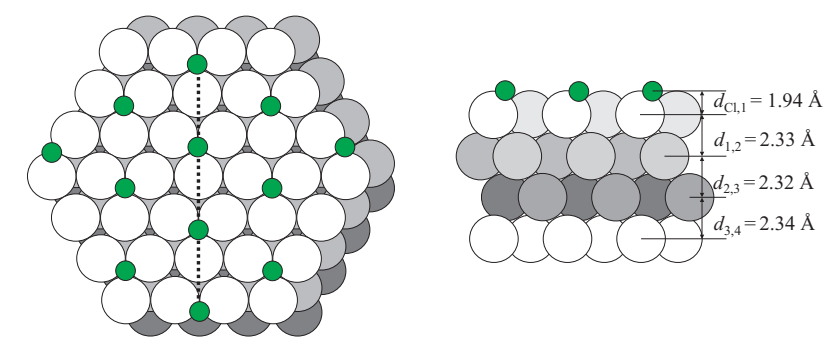

Fig. 3 Best-fit model of $\mathrm{Au}\{111\}-p(\sqrt{3} \times \sqrt{3}) R 30^{\circ}$-Cl. Left: top view, right: side view, cut along the $[11 \overline{2}]$ direction indicated by the dashed line in the top-view drawing. The $\mathrm{Cl}$ atoms are located in $\mathrm{fcc}$ adsorption sites (above the third layer gold atoms).

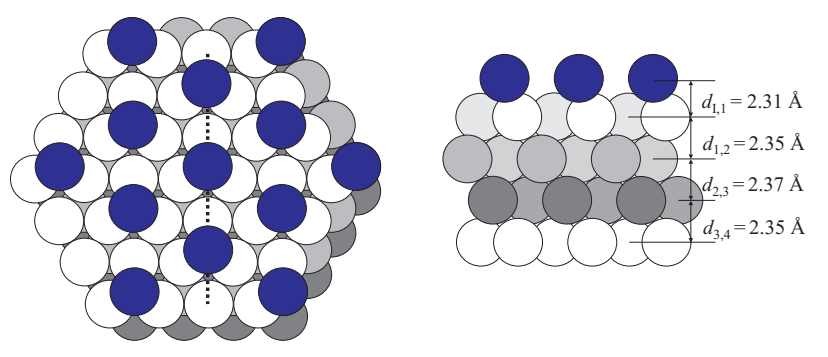

Fig. 4 Best-fit model of $\mathrm{Au}\{111\}-p(\sqrt{3} \times \sqrt{3}) R 30^{\circ}$-I. Left: top view, right: side view, cut along the $[11 \overline{2}]$ direction indicated by the dashed line in the top-view drawing. The I atoms are located in hcp adsorption sites (above the second layer gold atoms).

This geometry yields a minimum $R_{P}$ factor of 0.26 , which is significantly higher than that for the best fit $\mathrm{Cl}$ structure. Partly this is due to saturation of the LEED screen for high-intensity spots, which leads to plateau-like maxima for some energies in the experimental IV curves, which are not well reproduced in the theoretical curves. Still, the $R_{P}$ value of 0.26 is very significantly lower than the values for any other geometry model tested in this study (cf Table 1) and within the range of previous LEED-IV studies of halogen adsorption, with $R_{P}$ factors up to 0.27 reported $^{32}$. The atom positions within the best fit $\mathrm{Au}\{111\}-p(\sqrt{3} \times \sqrt{3}) R 30^{\circ}$-I surface structure are listed in Table 2 and depicted in Figure 4; for the experimental and simulated IV curves see Figure 5.

All relaxations of the top-most three Au layers with respect to the bulk positions are within the error bars of the structure determination but overall there is an expansion of these layers with respect to the chlorine structure. The iodine adatoms are bonded to the gold atoms surrounding the threefold hop sites at a vertical height of $2.31 \AA$, which corresponds to I-Au bond lengths of $2.84 \AA$. This is only $0.05 \AA$ larger than the sum of the covalent radii of gold and iodine (1.44 $\AA$ and
$1.35 \AA$ ) and $0.06 \AA$ smaller than the bond length of $2.90 \AA$ predicted theoretically ${ }^{19}$ but very different from the bond length, $2.62 \AA$, in the linear chain of AuI ${ }^{35}$. As I is less electronegative than the $\mathrm{Cl}$ one would expect a more covalent bond between $\mathrm{Au}$ and I and a bond length in accordance with findings of previous studies on chlorine adsorption on fcc transition metals that show metal-halogen bond lengths approximately equal to the sum of the covalent radii of halogen and metal

(a)
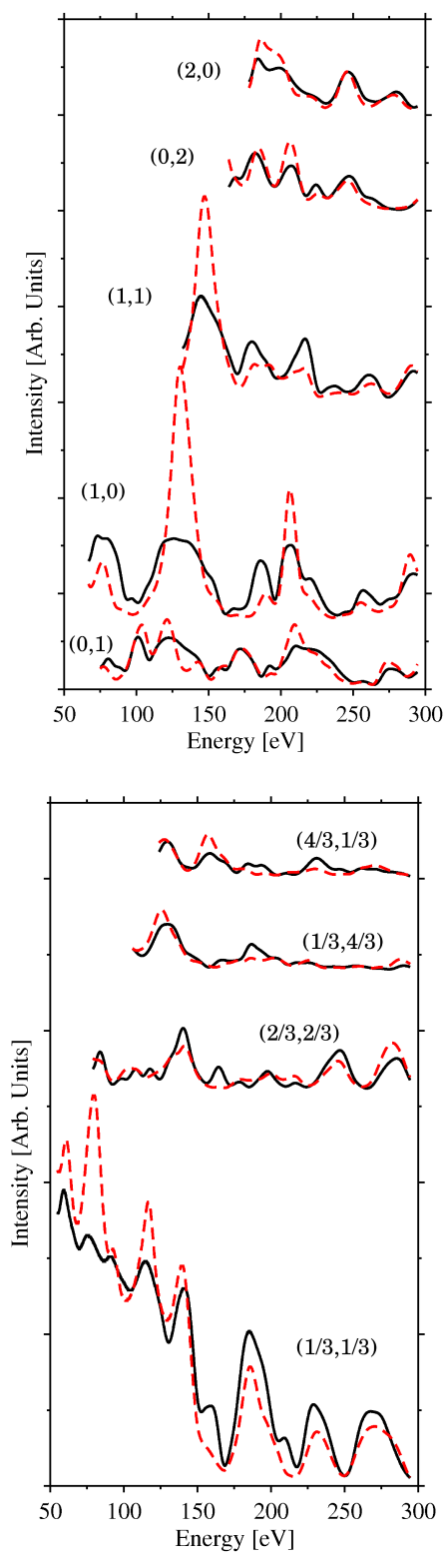

Fig. 5 Experimental (solid) and theoretical (dashed) LEED-IV curves for the best fit $\mathrm{Au}\{111\}-p(\sqrt{3} \times \sqrt{3}) R 30^{\circ}$-I structure: (a) integer (b) fractional order spots. 


\section{atoms $^{17,33,36-39}$.}

Strong ionic interaction should cause disturbance to the $\mathrm{Au}\{111\}$ substrate. We do not find, however, any strong relaxation with respect to the gold bulk structure in either of the two structures, which corroborates the covalent nature of the $\mathrm{Au}-\mathrm{Cl} / \mathrm{I}$ bonds. On the other hand, the fact that the surface layer of gold atoms is bulk-like is a very significant change with respect to the clean surface, where an uniaxially compressed " $(22 \times \sqrt{3})$ " herringbone reconstruction is observed, which has a $5 \%$ higher atom density than the bulk-terminated surface ${ }^{40}$. This surface reconstruction is due to a lack of bonds and, hence, a surplus of electrons in the surface layer and can be modified by changing the effective surface charge. Adsorbed alkali atoms cause the top layer of Au to become even more densely packed ${ }^{41}$, whereas electronegative atoms like $\mathrm{O}$, I, or $\mathrm{Cl}$ lift the herringbone reconstruction ${ }^{12,14,21,25,42}$. The excess gold atoms are incorporated into the $(1 \times 1)$ surface layer at the step edges. Previous STM studies showed that these structural transitions usually require less than stoichiometric amounts of adsorbate atoms ${ }^{14,41}$, indicating that it is indeed the charge transfer and not the bonding of individual atoms that causes the gold surface to transform.

For both chlorine and iodine, $a b$ initio calculations seem to systematically overestimate the $\mathrm{Au}-\mathrm{X}$ bond length. While this "underbinding" does probably not affect the conclusions about the covalent nature of the bond - in fact the experimental values are closer to those expected for a covalent bond - it may explain the discrepancies between theory and experiment in terms of adsorption sites preference. With the adsorbate being closer to the surface the energy difference between the hcp and fcc adsorption site should be more pronounced, since it is due to the arrangement of atoms in the second layer, and may even change its sign. All theoretical studies of halogen adsorption on $\mathrm{Cu}, \mathrm{Ag}$, or $\mathrm{Au}\{111\}$ surfaces find very small energy differences, of the order of a few $10 \mathrm{meV}$ per adsorbate atom, between hcp and fcc sites and a preference for fcc at low coverage around $0.33 \mathrm{ML}^{15,16,19,21,24}$. This would imply some degree of thermally induced site disorder, even at $150 \mathrm{~K}$, which has never been reported for $\mathrm{Cl}$ or $\mathrm{I}$ on $\mathrm{Au}\{111\}^{14,21}$. More strikingly, however, we find two different adsorption sites, fcc and hcp, respectively, for $\mathrm{Cl}$ and I in our experimental study, whereas $a b$ initio calculations predict fcc sites for both adsorbates.

\section{Summary}

A quantitative low-energy electron diffraction structural analysis has been performed for the ordered $\mathrm{p}(\sqrt{3} \times \sqrt{3}) R 30^{\circ}$ structures of chlorine and iodine on $\mathrm{Au}\{111\}$. Chlorine adsorbs on fcc hollow sites, at a vertical distance of $1.94 \AA$ which leads to a Cl-Au bond length of $2.56 \AA$. The top-most layers of the gold substrate are essentially bulk-like with small contractions of the first and second gold interlayer distances by up to $1.2 \%$. Iodine adatoms are bonded in hcp hollow sites at a vertical distance of $2.31 \AA$ from the first gold layer and a I-Au bond length of $2.84 \AA$ with no significant relaxation in the substrate with respect to the bulk positions. In both cases, the halogen-gold bonds are shorter than theoretically predicted and close to the sums of the covalent radii of the atoms involved, which corroborates the covalent nature of these bonds.

\section{Acknowledgement}

ZVZ acknowledges a postgraduate studentship grant from the "Research Endowment Trust Fund" of the University of Reading. We would also like to thank Dr R. A. Bennett for making the $\mathrm{Cl}$ evaporator available to us.

\section{References}

1 M. Haruta and M. Date, Applied Catalysis A, 2001, 222, 427 - 437.

2 M. Haruta and M. Date, Cattech, 2002, 6, $102-115$.

3 D. W. Goodman, J. Catal., 2003, 216, 213.

4 X. Y. Deng, B. K. Min, A. Guloy and C. M. Friend, Journal of the American Chemical Society, 2005, 127, 9267-9270.

5 D. S. Pinnaduwage, L. Zhou, W. Gao and C. M. Friend, Journal of the American Chemical Society, 2007, 129, 1872.

6 P. H. Citrin, P. Eisenberger, R. C. Hewitt and H. H. Farrell, Journal of Vacuum Science \& Technology, 1979, 16, 537.

7 N. D. Spencer and R. M. Lambert, Surface Science, 1981, 107, 237 - 248.

8 M. Maglietta, E. Zanazzi, U. Bardi, D. Sondericker, F. Jona and P. M. Marcus, Surface Science, 1982, 123, 141-151.

9 M. D. Crapper, C. E. Riley, P. J. J. Sweeney, C. F. McConville, D. P. Woodruff and R. G. Jones, Surface Science, 1987, 182, 213-230.

10 G. M. Lamble, R. S. Brooks, S. Ferrer, D. A. King and D. Norman, Physical Review B, 1986, 34, 2975-2978.

11 R. L. McCarley and A. J. Bard, Journal of Physical Chemistry, 1991, 95, 9618-9620.

12 G. N. Kastanas and B. E. Koel, Applied Surface Science, 1993, 64, 235 249.

13 B. M. Ocko, G. M. Watson and J. Wang, Journal of Physical Chemistry, 1994, 98, 897-906.

14 L. Huang, P. Zeppenfeld, S. Horch and G. Comsa, Journal of Chemical Physics, 1997, 107, 585 - 591.

15 K. Doll and N. M. Harrison, Chemical Physics Letters, 2000, 317, $282-$ 289.

16 K. Doll and N. M. Harrison, Physical Review B, 2001, 63, 165410.

17 A. G. Shard, C. Ton-That, P. A. Campbell and V. R. Dhanak, Physical Review B, 2004, 70, 155409.

18 A. Tkatchenko and N. Batina, Journal of Physical Chemistry B, 2005, 109, 21710-21715.

19 A. Migani and F. Illas, Journal of Physical Chemistry B, 2006, 110, 11894-11906.

20 B. V. Andryushechkin and K. N. Eltsov, Physics of Wave Phenomena, 2007, 15, $207-217$.

21 W. Gao, T. A. Baker, L. Zhou, D. S. Pinnaduwage, E. Kaxiras and C. M. Friend, Journal of the American Chemical Society, 2008, 130, 35603565 .

22 T. A. Baker, C. M. Friend and E. Kaxiras, Journal of the American Chemical Society, 2008, 130, 3720 - 3721 . 
23 T. A. Baker, C. M. Friend and E. Kaxiras, Journal of Chemical Physics, 2008, 129, 104702.

24 T. A. Baker, C. M. Friend and E. Kaxiras, Journal of Physical Chemistry, 2009, 130, 084701

25 S. A. Cochran and H. H. Farrell, Surface Science, 1980, 95, 359 - 366.

26 G. Held and W. Braun, CLEED manual, available from the authors.

27 J. B. Pendry, Low Energy Electron Diffraction, Academic Press, London, 1974.

28 M. A. Van Hove and S. Y. Tong, Surface Crystallography by LEED, Springer, Berlin, 1979.

29 A. Barbieri and M. A. Van Hove, Phase shift program package, available from http://electron.lbl.gov/software/software.html.

30 W. H. Press, B. P. Flannery, S. A. Teukolsky and W. T. Vetterling, Numerical Recipes in C, Cambridge University Press, Cambridge, 1988.

31 J. B. Pendry, J. Phys., 1980, C 13, 937.

32 C. J. Barnes, A. Wander and D. A. King, Surface Science, 1993, 281, 33-41.

33 M. Saidy, K. A. R. Mitchell, S. A. Furman, M. Labayen and D. A. Harrington, Surface Review and Letters, 1999, 6, 871-881.

34 W. Braun, H.-P. Steinrück and G. Held, Z. Phys. Chem., 2004, 218, 915.

35 A. F. Holleman and E. Wiberg, Lehrbuch der anorganischen Chemie, Walter de Gruyter, Berlin, 101st edn, 1995.

36 F. Forstmann, Japanese Journal of Applied Physics, 1974, 657-659.

37 A. G. Shard, V. R. Dhanak and A. Santoni, Surface Science, 1999, 429, $279-286$.

38 V. R. Dhanak, A. G. Shard, S. D'Addato and A. Santoni, Chemical Physics Letters, 1999, 306, 341-344.

39 A. G. Shard, V. R. Dhanak and A. Santoni, Surface Science, 2000, 445, $309-314$.

40 J. V. Barth, H. Brune, R. J. Behm and G. Ertl, Physical Review B, 1990, 42, 9307 - 9318 .

41 J. V. Barth, R. J. Behm and G. Ertl, Surface Science, 1994, 302, L319L324.

42 K. D. Gibson and S. J. Sibener, Journal of Physical Chemistry A, 2007, 111, 12398-12401.

\section{TOC Figure}
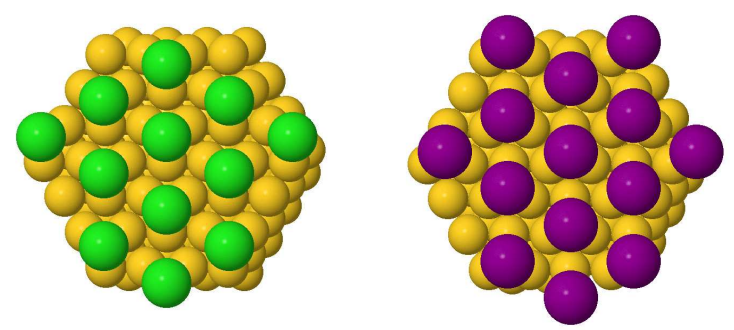

The experimental structure of I and $\mathrm{Cl}$ on $\mathrm{Au}\{111\}$ shows different adsorption sites and bond lengths significantly shorter than theoretically predicted.

\begin{tabular}{|c|c|c|c|c|c|c|}
\hline 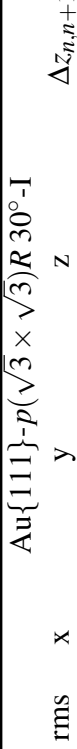 & $\begin{array}{l} \pm \\
0 \\
\dot{0} \\
+1 \\
\infty \\
m \\
0\end{array}$ & 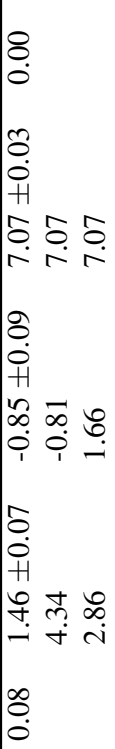 & 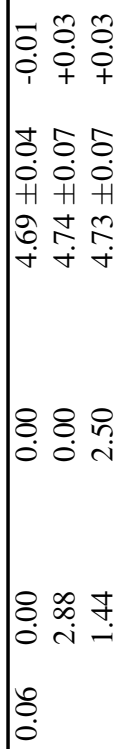 & 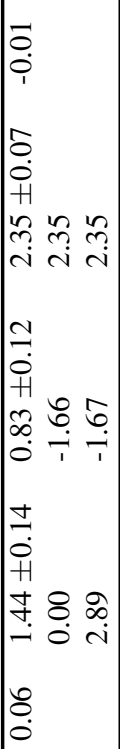 & ڤִ & 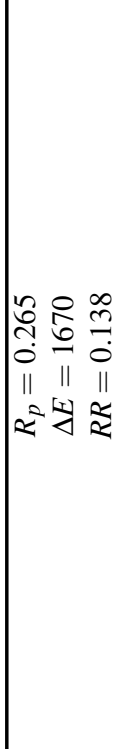 \\
\hline 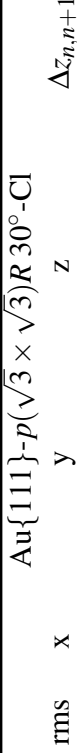 & 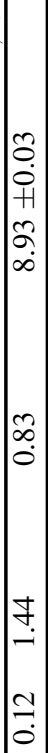 & 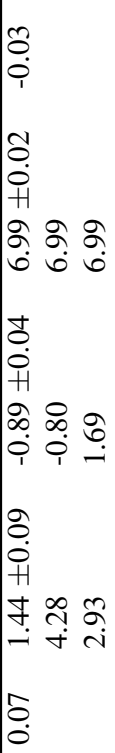 & 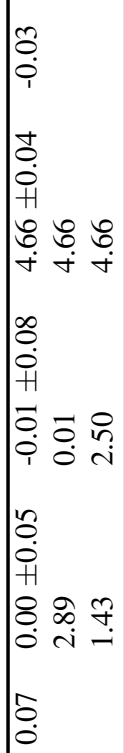 & 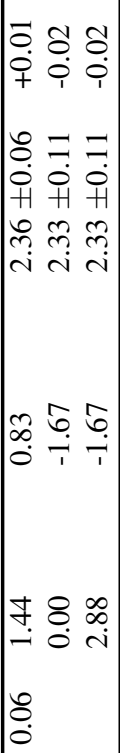 & ڤִ & 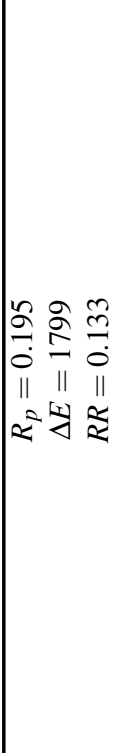 \\
\hline 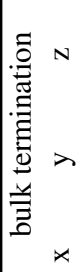 & & 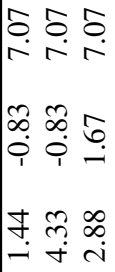 & 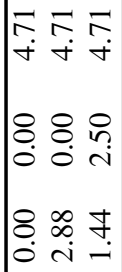 & 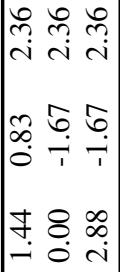 & $\begin{array}{l}8 \\
0 \\
0 \\
\infty \\
\infty \\
0 \\
1 \\
\dot{\theta} \\
\dot{+}\end{array}$ & \\
\hline & $\bar{\Xi}$ & $=\overrightarrow{\overline{0}}$ & $=\stackrel{N}{\stackrel{0}{D}}$ & $=\stackrel{m}{\stackrel{m}{\bar{D}}}$ & 至 & \\
\hline
\end{tabular}

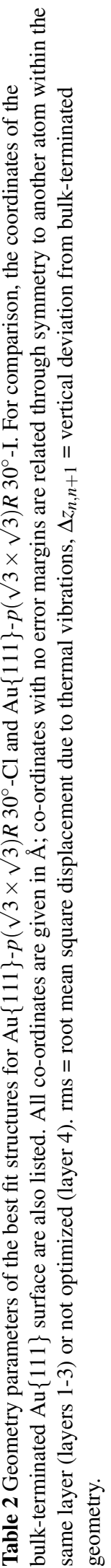

